\title{
„Faccend'ogni Toscan di te tremare“: Resonanzen politischer Kontroversen in Florentiner Liedsätzen
}

\section{$\operatorname{um} 1400$}

\author{
Signe Rotter-Broman
}

Zwischen 1300 und 1430 etabliert sich in Italien eine neue Form spätmittelalterlicher Liedkunst, die von Musikhistorikern gemeinhin als Kernrepertoire einer eigenständigen Epoche namens Trecento oder Ars nova Italiana verstanden wird. ${ }^{1}$ Das damit abgesteckte Repertoire besteht aus mehrstimmig, genauer: aus in zwei beziehungsweise drei Stimmen überlieferten weltlichen Liedsätzen. $^{2}$ Deren Texte wurden gezielt für die Vertonung verfasst; schon die Zeitgenossen verstanden diese Gedichte als Poesia per musica. ${ }^{3}$ Die primären poetisch-musikalischen Gattungen sind in der Spätphase des Trecento vor allem Madrigal und Ballata. Die musikalischen Quellen, mindestens sieben erhaltene, zum Teil umfangreiche Handschriften ${ }^{4}$ sowie eine erhebliche und durch jüngste Entdeckungen stetig anwachsende Zahl von Fragmenten, stammen - bis auf eine Ausnahme - aus der Zeit um und nach 1400. Sie zeichnen die Musik nach Tonhöhe und Rhythmus präzise in Mensuralnotation auf. Aufgrund der besagten Ausnahme, des erst in den 192oer Jahren entdeckten Codex Rossi (ca. 1350), ${ }^{5}$ konnte man die eigenständige Ausprägung dieser mehrstimmigen Kompositionsweise schon für die Mitte des 14. Jahrhunderts belegen.

1 Vgl. Baumann (1999).

2 Erst in jüngster Zeit ist der Blick auch auf weltliche Motetten und geistliche Kompositionen, insbesondere auf einzelne Messensätze, gerichtet worden.

3 Ziino (1995) 455-458.

4 Florenz, Biblioteca Nazionale Centrale, Ms. Panciatichi 26 (FP), Faksimile: Gallo (1981); London, British Library, Additional 29987 (Lo), Faksimile: Reaney (1965); Lucca, Archivio di Stato, Ms. 184 und Perugia, Biblioteca comunale Augusta, Ms. 3065 (Luc), Faksimile: Nádas und Ziino (1990); Paris, Bibliothèque Nationale, Ms. nouvelle acquisition française 6771 (Codex Reina, PR); Paris, Bibliothèque Nationale, Ms. fonds it. 568 (Pit); Florenz, Biblioteca Medicea-Laurenziana, Ms. Mediceo-Palatino 87 (Sq), Faksimile: Gallo (1992); Florenz, Biblioteca Medicea-Laurenziana, Archivio Capitolare San Lorenzo Ms. 2211 (sL, Palimpsest).

5 Rom, Biblioteca Vaticana, Codex Rossi 215 / Ostiglia, Fondazione Opera Pia don Giuseppe Greggiati (Rs), Faksimile: Pirrotta (1992). 
Darauf gestützt hat die Forschung das Hauptgewicht der Epoche des Trecento auf die innovative Leistung der frühesten Komponistengenerationen gelegt. ${ }^{6}$ Der berühmte blinde Organist Francesco Landini, aktiv von etwa 136o bis 1390, wurde nach diesem Epochenbild bereits als zweiter und letzter Höhepunkt, quasi als Summator der italienischen Eigentradition, definiert. ${ }^{7}$ Was danach geschah, also in der Generation der um und nach 1400 aktiven Musiker, wurde hingegen aufgrund des in den Handschriften dokumentierten Konservierungsstrebens, aufgrund der Wiederaufnahme älterer Gattungsmodelle sowie aufgrund des zunehmenden, vielbeschworenen „französischen Einflusses“ zumeist mit Etiketten wie „Bewahrung der Tradition“, wenn nicht sogar „Konservativismus“ oder „Manierismus“ versehen. ${ }^{8}$

In der Tat spielen Modelle des frühen und mittleren Trecento für diese späte Komponistengeneration, insbesondere für die in Florenz ansässigen Musiker wie Paolo da Firenze oder Andrea da Firenze, eine wesentliche Rolle. Auf der Suche nach einer Erklärung dafür, gerade auch hinsichtlich der um 1400 gehäuft auftretenden Verwendung von Madrigaltexten mit politischem Inhalt, stützte man sich auf eine These des Kulturhistorikers Hans Baron, die ins Zentrum der Thematik dieses Sammelbands führt. Ich gebe die These in der prinzipiell zustimmenden Zusammenfassung von Gene Brucker wieder:

Die Ursprünge des bürgerlichen Humanismus seien in der politischen Situation Florenz' zur Zeit um 1400 zu suchen, noch genauer: in der Bedrohung der Unabhängigkeit der Stadt, verkörpert durch Giangaleazzo Visconti. Diese Krise hätte nachhaltigen Einfluss auf die Mentalität der Florentiner gehabt, sie bestärkte die Bürger in ihrer Treue zur republikanischen Regierung und zu den Idealen von Freiheit und Freizügigkeit, die traditionell mit dieser Regierungsform verbunden werden. ${ }^{9}$

Unter den Literaturwissenschaftlern hat vor allem Antonio Lanza die Auswirkungen der Konfrontation zwischen der Mailänder Signoria und der Republik

6 Eine weite Verbreitung erhielt diese von den um 1900 wirkenden ,Gründervätern' Johannes Wolf und Friedrich Ludwig ausgehende Sicht durch Besseler (1931). Weiterhin signifikant für das Epochenbild waren die Forschungen von Martínez [-Göllner] (1963) sowie die Schriften von Von Fischer, beispielsweise in seinem Aufsatz Von Fischer (1984). Zur heutigen Sicht auf die Forschungsgeschichte siehe Huck (2005).

7 Dies spiegelt sich etwa in den Beiträgen des von Barezzani und Delfino herausgegebenen Sammelbands, Barezzani und Delfino (1999), und in der jüngst erschienenen Biographie von Fiori (2004).

8 Vgl. dazu ausführlicher Rotter-Broman (2007).

9 Brucker (1990) 283; vgl. Baron (1955). 
Florenz um 1400 auf die literarische Produktion und das intellektuelle Klima in Florenz dieser Jahre untersucht. ${ }^{10}$ Als berühmte Beispiele literarischer Kontroversen werden von ihm etwa die Entgegnungen von Franco Sacchetti und Cino Rinuccini auf die Invectiva in Florentinos des Mailänder Humanisten Antonio Loschi angeführt.

Dieser Forschungsstand hat in der musikbezogenen Trecento-Forschung die These hervorgebracht, die als retrospektiv verstandenen Tendenzen "nach Landini“ seien als Teil dieser Selbstvergewisserung im Gefolge der politischen Zuspitzungen um 1400 zu betrachten. So schreibt der ausgewiesene Quellenkenner John Nádas dem um 1400 wirkenden Komponisten Paolo da Firenze in seinen Madrigalen ein ausgeprägtes ,antiquarisches“ Bestreben zu, was sich mit der Nähe zum Florentiner Studio und seiner angenommenen Verantwortlichkeit für die Redaktion des Codex Squarcialupi, der prachtvollsten Trecentohandschrift, decke. ${ }^{11}$

Aus meiner Sicht erheben sich allerdings zwei Fragen. Zum einen wäre nicht vorauszusetzen, sondern erst zu belegen, inwieweit ein Bezug auf ältere Modelle als Streben nach Bewahrung des Alten, also als „Traditionalismus“ zu interpretieren ist. Weiterhin wäre zu überlegen, welche Verständnismöglichkeiten sich eröffnen, wenn die unmittelbare Textaussage nicht an sich, sondern vielmehr gemeinsam mit ihrer spezifisch lyrischen Verfasstheit in den Blick genommen wird. Bei Liedsätzen wäre daher nach der Wechselwirkung von lyrischer Form und musikalischer Form zu fragen. Das so umrissene Beziehungsnetz wird schließlich durch die Umsetzung des Gedichttexts in mehreren Stimmen zusätzlich potenziert.

Der vorliegende Beitrag widmet sich diesen Fragen am Beispiel des dreistimmigen Madrigals Godi Firenze von Paolo da Firenze. ${ }^{12}$ Paolos Madrigal lässt sich auf 1406 datieren; ${ }^{13}$ der Text feiert den endgültigen Sieg von Florenz über die Nachbarstadt Pisa. Diesem Sieg waren verwickelte, ebenso mit Waffen wie mit

$10 \quad$ Lanza $(1991,1994)$.

11 „A sense of intentionally restoring an artistic heritage by both intellectual and musicalpoetic circles is particularly well reflected in the most elusive, but certainly the outstanding, composer/poet of the period - Paolo Tenorista." Nádas (1990) 131. Vgl. auch die Formulierung „Paolo's antiquarian tastes“ auf S. 133. Siehe ferner Nádas (1989).

12 Paolo da Firenze (ca. 1355-1436) gehörte als höherrangiger Amtsträger innerhalb des Benediktinerordens der Florentiner klerikalen Elite an; seine Teilnahme am Konzil von Pisa 1409 ist dokumentiert. Nach heutigem Stand stellt der von ihm überlieferte Liedsatzbestand den zweitgrößten des Trecento nach Francesco Landini dar. Eine verlässliche Darstellung des Forschungsstands bietet Fallows (2001).

13 Verlässliche Edition: Günther (1967) 115-119. Weitere Editionen (z.T. fehlerhaft): Marrocco (1975) und Monterosso (1966) 188-193. 
politischen Winkelzügen geführte Auseinandersetzungen vorausgegangen, die sich im Spannungsfeld der politischen Mächte Florenz und Mailand mit dem Machtzentrum Frankreich im Hintergrund abspielten. ${ }^{14}$

Die einzige Quelle für Godi Firenze ist eine in Florenz entstandene Handschrift, die heute in der Pariser Bibliothèque Nationale aufbewahrt wird. ${ }^{15}$ Der Text lautet:
Godi, Firenze, po'che se' sì grande che batti l'ale per terr'e per mare, Faccend'ogni Toscan di te tremare. Glorioso triunfo di te spande per tutto l'universo immortal fama, po'che Pisa tuo serva omai si chiama. Giove superno e'l Batista di gloria dànno di Pisa al tuo popol vittoria. ${ }^{16}$

Schon der erste Vers ruft die Kategorie „Traditionsbezug“ auf, treibt der unbekannte Verfasser doch ein unüberhörbares intertextuelles Spiel mit Dantes Commedia, genauer mit den ersten drei Versen der sechs beginnenden Verse des 26. Gesangs im Inferno: ${ }^{17}$
Godi Fiorenza poiché se' sì grande che per mare e per terra batti l'ali e per lo'nferno tuo nome si spande! Tra li ladron trovai cinque cotali Tuoi cittadini onde mi ven vergogna, e tu in grande orranza non ne sali. ${ }^{18}$

\footnotetext{
14 Günther (1967) 100-102.

15 Paris, Bibliothèque Nationale, Ms. fonds it. 568 (Pit), $56^{\mathrm{v}}-57^{\mathrm{r}}$.

16 Corsi (1970) 269. Übersetzung der Verfasserin:, Freu dich, Florenz, denn du bist ja so groß, dass du die Flügel schlägst über Land und Meer und jeden Toscaner vor dir erzittern lässt. Der glorreiche Sieg verbreitet im ganzen Universum deinen unvergänglichen Ruhm, denn Pisa nennt sich nun auf immer deine Dienerin. Jupiter und [Johannes] der Täufer geben deinem Volk den Sieg über Pisa.'

17 Monterosso (1966) 185.

18 Inf. XXVI, 1-3. Sapegno (1957) 295. Vgl. für Paolos Verweise auf Dante Pirrotta (1961) 2829. Übers. Naumann (2003), das Zitat aus Bd. 2 (Erläuterungsband), 144; die Übersetzung aus Bd. 1, 137: ,Frohlocke, Florenz, denn so groß bist du, dass über Land und Meer du die Flügel schlägst und in der Hölle sich dein Name ausbreitet. Unter den Dieben fand ich von deinen Bürgern fünf solche, dass mich Scham darüber ankommt und es dir nicht zu großer Ehre gereicht:
} 
Doch handelt es sich hierbei tatsächlich um eine "Auctoritas Dantesca“, wie Raffaello Monterosso 1966 postulierte? ${ }^{19}$ Immerhin weckt die Anspielung auf Dante zunächst nicht unbedingt erbauliche Assoziationen. Denn der 26. Gesang des Inferno behandelt den zweifelhaften Ruhm, den Florenz kraft seiner Diebe und Gauner in der Unterwelt genießt. „Im Rückblick auf die Begegnung mit den fünf Dieben im siebten Graben", so kommentiert Walter Naumann, „lässt Dante seinen dunklen Gefühlen und Ahnungen für seine Vaterstadt in einem sarkastischen Ausruf ,Frohlocke!' [Godi] freien Lauf." ${ }^{\text {20 }}$

Dass sich Paolo aus politischen Motiven auf das Dante-Zitat nur als Auctoritas, unbekümmert um diese Bedeutungsdifferenz, bezogen habe, ${ }^{21}$ scheint angesichts seiner umfassenden Bildung und der von Monterosso selbst angenommenen Dante-Textkenntnis eher unwahrscheinlich. Viel eher scheint sich dieser Bedeutungswandel in eine generelle Tendenz einzufügen, die um 1400 als Madrigal-spezifisches Spiel mit Referenzen weit verbreitet ist. Die Anspielung auf Dante wird durch ihre inhaltliche und formale Umsetzung ausschlaggebend für die Anlage des musikalischen Satzes in seiner zeitlichen Struktur.

Paolos Madrigaltext (der Textdichter ist unbekannt, es könnte sich aber durchaus um den Komponisten selbst handeln ${ }^{22}$ weist das Reimschema abb acc dd auf. Die zwei Terzinen werden also durch die gleiche Reimsilbe in der ersten Zeile verkoppelt. Wie bei dieserart intertextuellen Anspielungen üblich, lässt Paolo das Gedicht nach dem capoverso-Zitat - scheinbar - seine eigene Wendung nehmen („Che batti l'ale“ statt "che per mare e per terra“), doch erweist sich trotz syntaktischer Umstellung anhand der beibehaltenen Begriffe, dass auch die zweite Verszeile mehr oder weniger vollständig auf Dante zurückgeht. Allerdings liefert nicht das Wort l'ali, sondern mare die Reimsilbe. In Paolos drittem Madrigalvers wäre nun analog zu Dantes abaTerzine ebenfalls ein a-Reim zu erwarten, doch diese Erwartung wird mit dem homonymen b-Reim ,mare - tremare unüberhörbar enttäuscht. Erst ab dem dritten Vers also wird reimstrukturell - und zugleich inhaltlich - der von Dante abweichende Inhalt hervorgehoben: „du lässt jeden Toscaner vor dir zittern".

Allerdings bleibt hier noch offen, ob das Zittern der Toscaner mit Dante ironisch auf die zwielichtige Welt der Hölle zu beziehen ist. Erst mit dem

\footnotetext{
19 Monterosso (1966) $185 \mathrm{f}$.

$20 \quad$ Naumann (2003) Bd. 2, 144.

21 Monterosso (1966) 187.

22 Pirrotta (1961) 28.
} 
ersten Vers der zweiten Strophe wird unmissverständlich klargestellt, dass statt der zweifelhaften Verbreitung des schlechten Rufs durch die Hölle nun von der Verkündung des Florentiner Triumphs durch das ganze Universum die Rede ist ("glorioso triunfo di te spande“ statt "per lo'nferno tuo nome si spande").

Der reimstrukturelle Bezug zu Dante verstärkt das gespannte Netz aus Anlehnung und Abweichung. So beendet beispielsweise spande bei Dante die erste Terzine, während es bei Paolo zu Beginn der zweiten Strophe keine innerstrophische, sondern eine strophenübergreifende Funktion hat. Während der Reim spande bei Dante schließt, bildet er bei Paolo die Voraussetzung für die doppelpunktartige Öffnung des Gedichts zum Ritornell hin. Erst ab der dritten Zeile der ersten und vor allem im Verlauf der zweiten Strophe erhält Paolos Madrigaltext eine intern begründete, finalisierende Dynamik.

Wie setzt Paolo nun musikalisch diese - vom Text gerade auch durch strukturelle Merkmale getragene - Verlaufsdynamik um? Hier ist zuerst auf ein Merkmal der Eröffnung einzugehen, das auch ohne detaillierte Kenntnisse dieses Repertoires direkt ins Auge beziehungsweise ins Ohr springt: die mehrfache Wiederholung des Wortes Godi („Freu dich“) in allen Stimmen vor Beginn der eigentlichen Deklamation (Abb. 10.1). ${ }^{23}$

Diese Exklamation wurde von der Forschung zumeist als klares Indiz dafür gelesen, dass der Musik eine den Textinhalt abbildende Funktion zukomme, so meint etwa Monterosso, dass der Beginn eine besondere Beachtung verdiene, da bei diesem in allen drei Stimmen eine realistisch-beschreibende Absicht in der rhythmischen Struktur offensichtlich sei. Diese unterstreiche mit der Deklamationsenergie die Erleichterung im Godi. ${ }^{24}$

Doch lässt sich dieses Phänomen in Hinblick auf die Gattungstradition auch aus einem anderen Blickwinkel verstehen. Denn zu den für Paolos Kompositionsweise charakteristischen Eigenheiten gehört das Spiel mit musikalischen Zeitstrukturen unabhängig von der deklamatorischen Zeit. ${ }^{25}$ Mit der durchbrochenen Setzweise (der zeitgenössische Terminus dafür lautet Hoquetus, also "Schluckauf“) steht paradoxerweise ein musikalisches Schluss-Signal

\footnotetext{
23 Günther (1967) 115.

24 Vgl. Monterosso (1966) 187. Die Einspielung des Satzes durch das Ensemble Mala Punica unter Leitung von Pedro Memelsdorff (Narcisso speculando. Madrigaux de Paolo da Firenze, Mala Punica/Pedro Memelsdorff, Harmonia mundi France 2002, HMC 901732) unterstreicht dieses realistisch-bildliche Verständnis durch die Verwendung von Fanfaren im Hoquetus und die prägnante sängerische Heraushebung von Phrasen wie che battil'ale oder tremare.

25 Fragen der Zeitgestaltung in Madrigalen und Ballaten bilden einen Schwerpunkt der
} 

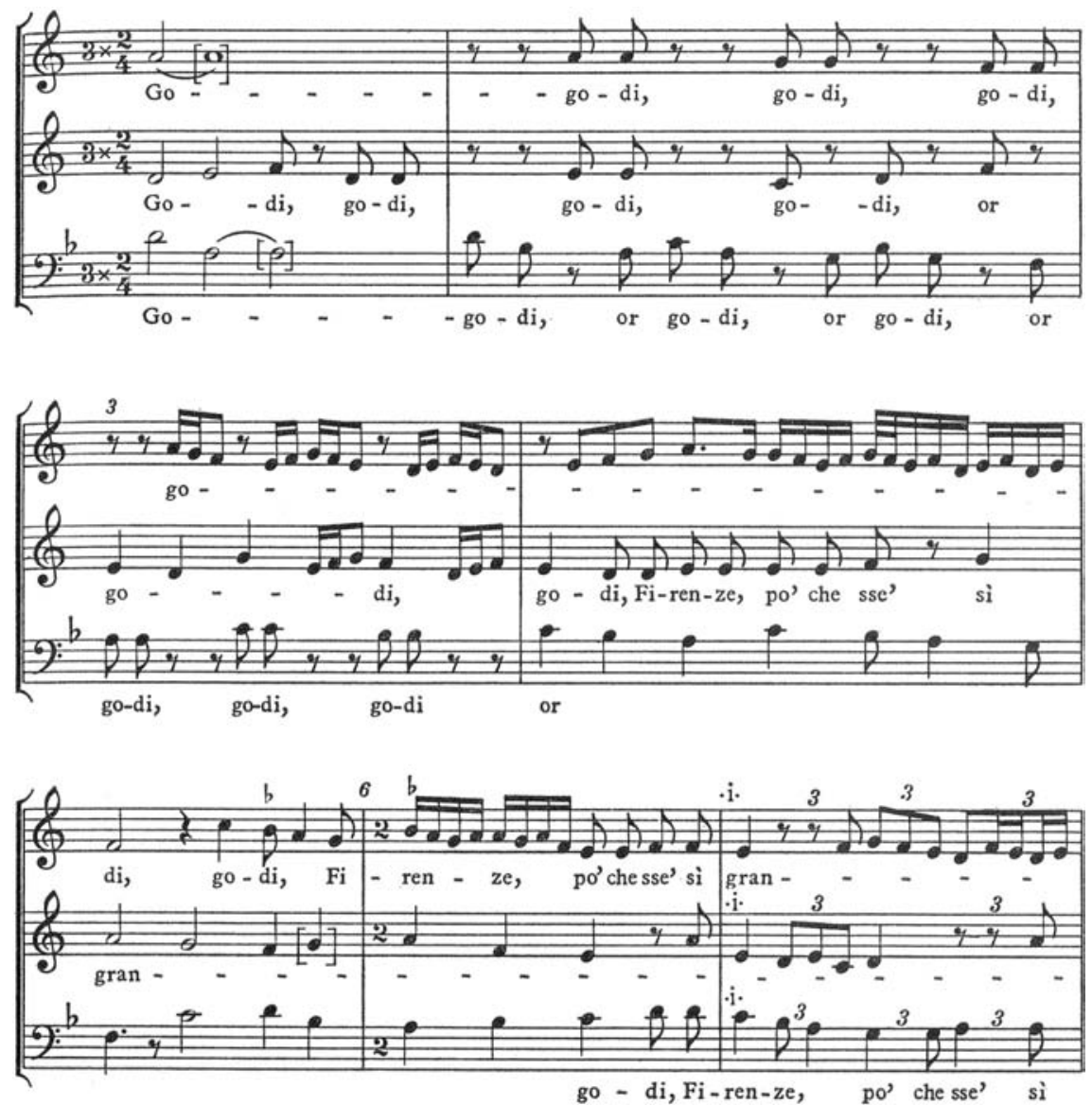

Авв. 10.1 Paolo da Firenze, Godi Firenze, ed. Günther, T. 1-7

am Anfang des Madrigals; Hoqueti erscheinen normalerweise in der Oberstimme bei der Penultima-Silbe in der abschließenden Zeile eines musikalischen Abschnitts. Mit dem Godi-Hoquetus verzögert Paolo also den gattungsgemäß „richtigen“ deklamierenden Einsatz der Stimmen nach hinten, denn aus den Godi-Rufen baut sich in den einzelnen Stimmen erst allmählich die Art von Kontinuität auf, die Voraussetzung für die geordnete Deklamation ist. Die „durch die Stimmen gereichte“, zeitlich versetzte Deklamation des ersten Verses verzögert die eigentlich zu erwartende Synchronizität von Musikzeit und

Untersuchungen in meiner Habilitationsschrift (Kiel 2009): Rotter-Broman (2012). Für eine exemplarische Darstellung der Verfahrensweise siehe Rotter-Broman (2008). 

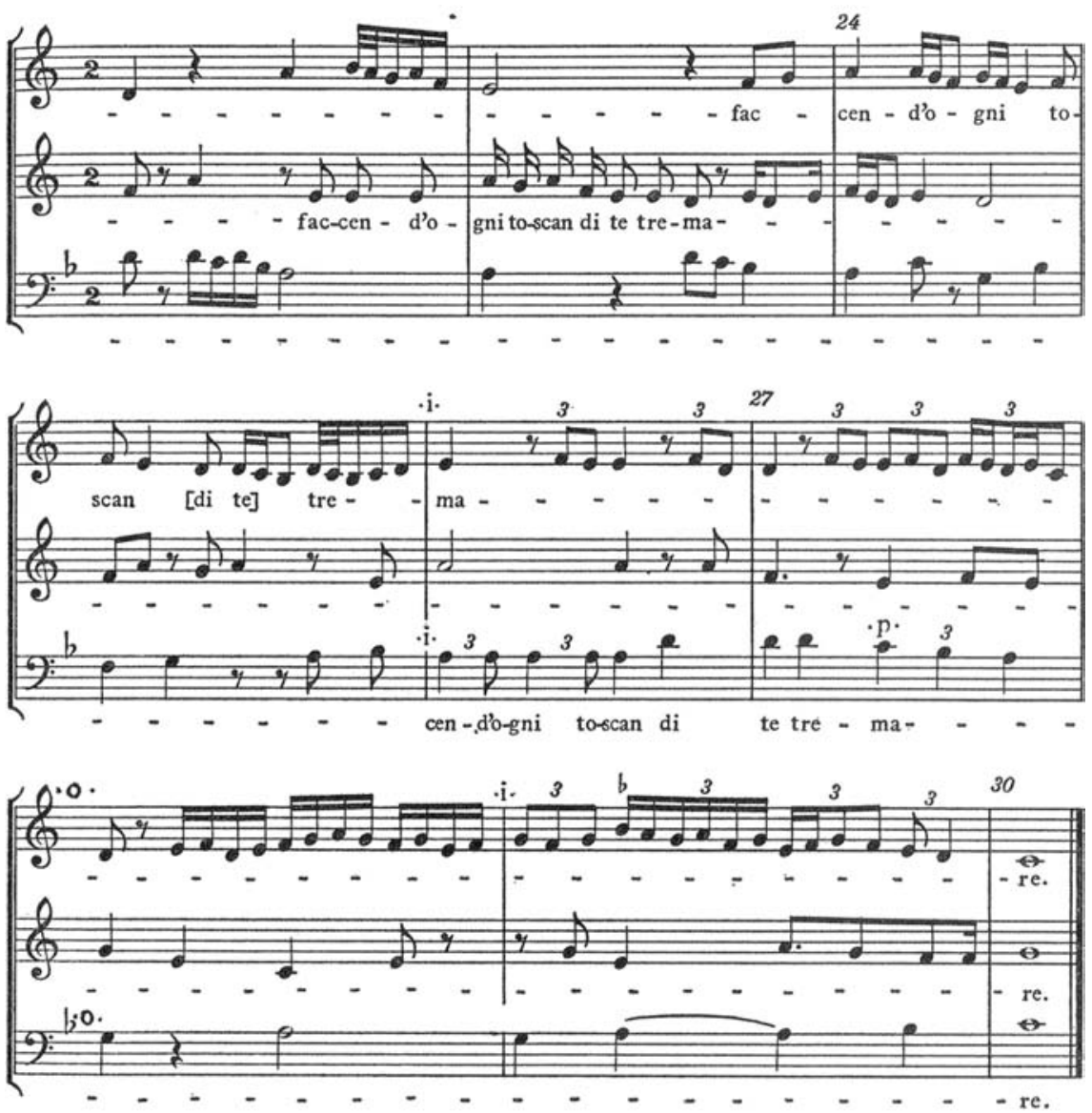

Авв. 10.2A Paolo da Firenze, Godi Firenze, ed. Günther, Ende der Strophe (T. 22-30)

Textzeit ebenfalls, denn die wichtigste Stimme, der Cantus, setzt erst im sechsten Takt mit seiner vollständigen Zeilendeklamation ein.

Ich erwähne nur mit Stichworten einige weitere Maßnahmen zur Verselbständigung der musikalischen Zeit, für die im Rahmen dieses Beitrags auf detaillierte Belege am Notentext verzichtet sei. ${ }^{26}$ Mehrmals durchkreuzt etwa die dritte Stimme (der sogenannte Contratenor) melodische Ruhepunkte (Kadenzen) der Außenstimmen (Cantus und Tenor). Die Cantus-Melodik kommt mitunter erst nach mehreren "Anläufen“ ins Ziel, und die Deklamation des zentralen dritten Verses „Faccend'ogni Toscan di te tremare“ wird in

26 Für eine ausführlichere analytische Darstellung siehe Rotter-Broman (2012) Kap. IV. 4: Die Madrigale. 

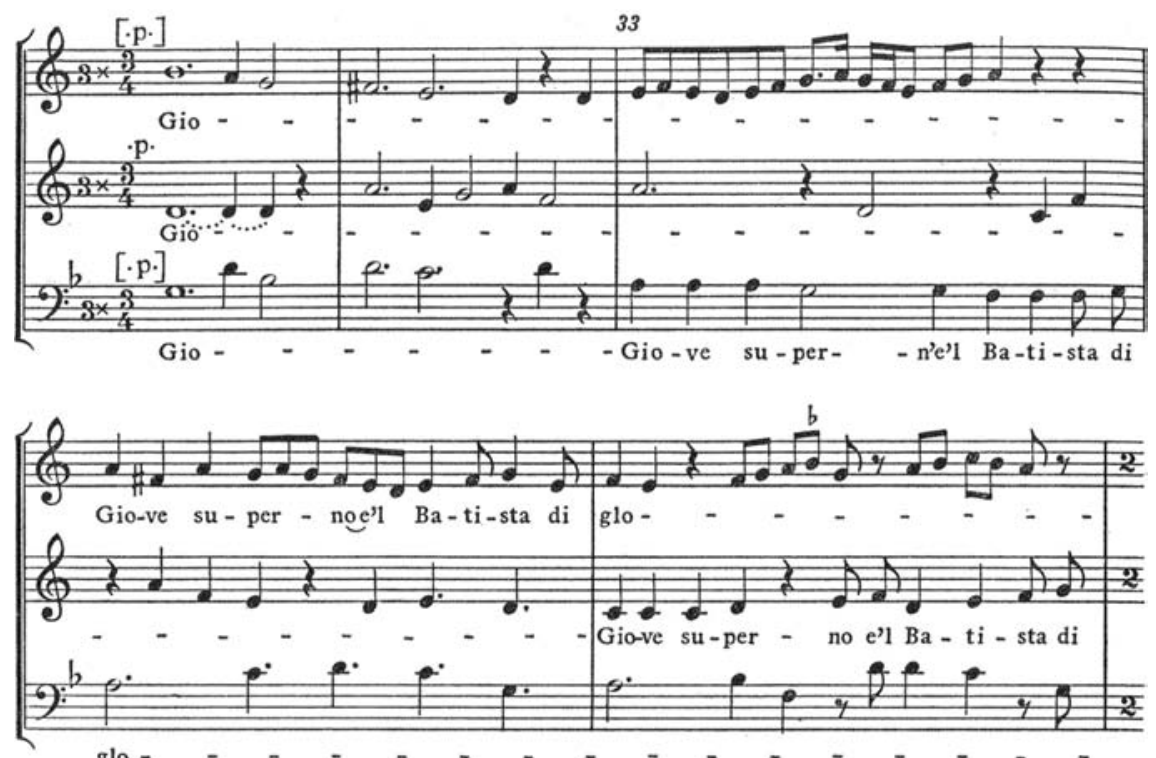

glo - - - - - - - - - - - - - - - -

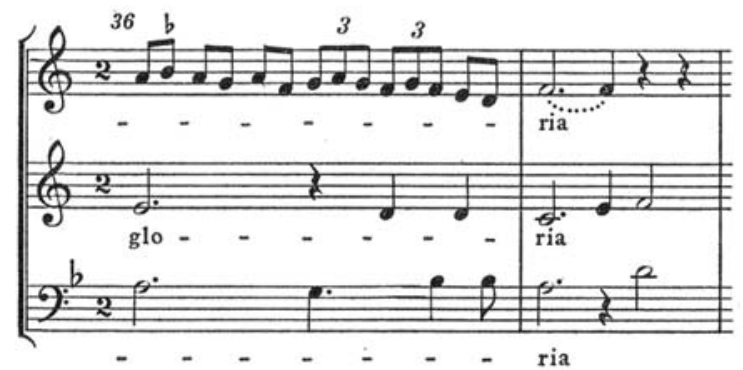

Авв. 10.2 в Paolo da Firenze, Godi Firenze, ed. Günther, Begin des Ritornells (T. 31-37)

Takt 21 durch besonders virtuose Linienführung im Cantus vorbereitet, wobei dieser dann doch noch in Takt 22 den Textvortrag des Contratenors abwarten muss, bevor er die gewichtige dritte Strophenzeile bis zur Penultima deklamiert.

Wesentlich ist der Aspekt, dass Paolo die Musikzeit getrennt von der Textzeit, also der Deklamationszeit, behandelt. Gerade damit unterstützt er im Großen die Struktur und Verlaufsdynamik des Textes in dessen Absetzungsbewegung vom Dante-Modell.

Auf einen letzten, sehr eindrücklichen Moment sei anhand der obigen Abbildungen noch hingewiesen, nämlich auf den Übergang zwischen Strophenteil und Ritornell. Zwar wird in den Schlusstakten des Strophenteils das Ritornell durchaus zielstrebig angesteuert (Abb. 10.2A), doch sein eigentlicher 
Beginn wird mit einer Art Zeitstau markiert (Abb. 10.2B). ${ }^{27}$ Die Maßnahme einer über längere Zeit beibehaltenen ersten Silbe entspricht zwar den Gattungsnormen, aber die grundsätzliche, abrupte Außerkraftsetzung der bisherigen rhythmischen Gangart zusammen mit den nach damaligem Maßstab viel zu lang gehaltenen „unvollkommenen“ Klängen (Terzen, Sexten) ohne Auflösung zu „vollkommenen“ Konsonanzen (Quinte, Oktave) ist wiederum als Dokument der Komponisten unabhängig vom Text gestalteter musikalischer Eigenzeit zu lesen. Die Hauptfunktion dieses Zeitstaus ist denn auch weniger, wie man vielleicht beim ersten Blick auf den Text assoziieren könnte, eine religiös angehauchte Reverenz an Jupiter, sondern eher eine Art Nullstellung der Musikzeit, aus der erneut die drei Stimmen allmählich ihre deklamatorische Identität erreichen, wiederum mit deutlicher hierarchischer Abstufung (Cantus - Tenor - Contratenor). Und zwischen den beiden gleichen Reimsilben des Textes (gloria - vittoria) differenziert die Musik deutlich im Sinne einer Finalisierung: unvollkommener Klang am Ende der ersten Zeile (Takt 37); vollkommener Klang mit Tenor und Cantus im Einklang in der zweiten Zeile.

Somit lässt sich schließlich zusammenfassen, dass für den Umgang der Musiker um 1400 mit den Modellen des mittleren Trecento die Verselbständigung musikalischer Zeitstrukturen kennzeichnend ist. Godi Firenze erscheint, in diesem Lichte betrachtet, weniger als auditive Illustration konfliktreicher Realität, sondern vielmehr als ein kunstvolles Spiel mit gattungsbezogenen Erwartungen, bei dem die Musik als strukturell bedeutungsvolle, eigengewichtige Ebene eingesetzt wird. Dies wird erreicht, indem auf Elemente ihrer eigenen Geschichte angespielt wird. Diese dienen somit nicht als bindende Modelle, sondern stehen als Material für eine um 1400 sich ausbreitende Tendenz zur selbstreflexiven Anreicherung des Tonsatzes zur Verfügung. Dem gezielten Spiel mit Nähe und Distanz zu Dante, das die Textvorlage kennzeichnet, entspricht in Paolos musikalischer Gestaltung die kalkulierte Reflexion tradierter kompositorischer Verfahrensweisen.

\section{Literatur}

Barezzani, M.T.R., und A. Delfino (Hg.) 1999. „Col dolce suon che da te piove“: studi su Francesco Landini e la musica del suo tempo in memoria di Nino Pirrotta. Florenz.

Baron, H. 1955. The Crisis of the Early Italian Renaissance. Civic Humanism and Republican Liberty in an Age of Classicism and Tyranny. 2 Bde. Princeton.

27 Dies setzt die Aufnahme mit Pedro Memelsdorff (vgl. Anm. 2424) sehr sensibel um. 
Baumann, D. 1999. Art. Trecento und Trecentohandschriften. In Die Musik in Geschichte und Gegenwart, hgg. L. Finscher, 2. Aufl., Sachteil Bd. 9, Sp. 769-791. Kassel/Stuttgart.

Besseler, H. 1931. Die Musik des Mittelalters und der Renaissance. Potsdam.

Brucker, G. 1990. Florenz in der Renaissance. Stadt, Gesellschaft, Kultur, engl. Orig.: Renaissance Florence (1969), übers. von Claudia Preuschoft. Reinbek.

Corsi, G. (Hg.) 1970. Poesie musicali del Trecento. Bologna (Collezione di opere inedite o rare 131 ).

Fallows, D. 2001. Paolo da Firenze. In The New Grove Dictionary of Music and Musicians, hgg. S. Sadie, 2. Aufl., Bd. 19. London: 49-51.

Fiori, A. 2004. Francesco Landini. Palermo (Constellatio musica 11).

Von Fischer, K. 1984. Das Madrigal ,Si com‘ al canto della bella Iguana' von Magister Piero und Jacopo da Bologna. In Analysen. Beiträge zu einer Problemgeschichte des Komponierens. Festschrift Hans Heinrich Eggebrecht, hgg. W. Breig u. a. Stuttgart: 4656.

Gallo, F.A. (Hg.) 1981. Il codice musicale Panciatichi 26 della Biblioteca nazionale di Firenze. Riproduzione in facsimile. Florenz (Studi e testi per la storia della musica 3).

Gallo, F.A. (Hg.) 1992. Il codice Squarcialupi. 2 Bde. (Faksimile/Kommentar). Florenz und Lucca.

Günther, U. 1967. Zur Datierung des Madrigals ,Godi, Firenze' und der Handschrift Paris, B.N., fonds it. 568. Archiv für Musikwissenschaft 25: 99-119.

Huck, O. 2005. Die Musik des frühen Trecento. Hildesheim u. a. (Musica mensurabilis 1).

Lanza, A. 1991. Firenze contro Milano. Gli intellettuali fiorentini nelle guerre con $i$ Visconti (1390-1440). Rom (Medioevo e Rinascimento 2).

Lanza, A. 1994. La letteratura tardogotica. Arte e poesia a Firenze e Siena nell'autunno del Medioevo. Rom.

Marrocco, T.W. 1975. Secular Trecento Music. Monaco 1975 (Polyphonic Music of the Fourteenth Century 9).

Martínez [Göllner], M.-L. 1963. Die Musik des frühen Trecento. Tutzing (Münchner Studien zur Musikgeschichte 9).

Monterosso, R. 1966. Un' ,Auctoritas' dantesca in un madrigale dell'Ars Nova. Collectanea Historiae Musicae 4: 185-193.

Nádas, J. 1989. The Songs of Don Paolo Tenorista: The Manuscript Tradition. In In cantu et in sermone: For Nino Pirrotta on His 8oth Birthday, hgg. F. Della Seta und F. Piperno. Florenz (Italian Medieval and Renaissance Studies 2): 41-64.

Nádas, J. 199o. Song collections in Late-Medieval Florence. In Atti delXIV congresso della società internazionale di musicologia: Trasmissione e recezione delle forme di cultura musicale, Bologna 27 agosto-1 ${ }^{\circ}$ settembre 1987, Ferrara-Parma, 30 agosto 1987, hgg. A. Pompilio u. a., Bd. 1: Round Tables. Torino: 126-137.

Nádas, J., und Ziino, A. (Hg.) 199o. The Lucca Codex (Codice Mancini). Lucca (Ars nova 1). 
Naumann, W. (übs.) 2003. Dante Alighieri, Die göttliche Komödie. 2 Bde. Darmstadt.

Pirrotta, N. (Hg.) 1961. Paolo Tenorista in a New Fragment of the Italian Ars Nova. A Facsimile Edition of an Early Fifteenth-Century Manuscript now in the Library of Professor Edward E. Lowinsky, Berkeley, California, with an Introduction by N.P. Palm Springs.

Pirrotta, N. (Hg.) 1992. Il codice Rossi 215/The Rossi Codex 215. Roma, Biblioteca Apostolica Vaticana/Ostiglia, Fondazione Opera Pia don Giuseppe Greggiati. Lucca (Ars nova 2). Reaney, G. (Hg.) 1965. The Manuscript London, British Museum, Additional 29987. A Facsimile Edition with an Introduction. Rom. (Musicological Studies and Documents 13)

Rotter-Broman, S. 2007. Geschichtsbild und Analyse. Überlegungen zur Musik des späten Trecento. In Kontinuität und Transformation in der italienischen Vokalmusik zwischen Due- und Quattrocento. Bericht über die Tagung in Jena vom 1.-3.Juli 2005, hgg. S. Dieckmann u.a. Hildesheim u. a. (Musica mensurabilis 3): 197-211.

Rotter-Broman, S. 2008. Musikzeit und Textzeit in Ballaten des späten Trecento. Die Tonkunst 2: 314-323

Rotter-Broman, S. 2012. Komponieren in Italien um 1400. Studien zu dreistimmig überlieferten Liedsätzen von Andrea und Paolo da Firenze, Bartolino da Padova, Antonio Zacara da Teramo und Johannes Ciconia. Hildesheim (Musica mensurabilis 6).

Sapegno, N. (Hg.). 1957. Dante Alighieri, La divina Commedia. Mailand und Neapel (La letteratura italiana 4).

Ziino, A. 1995. Rime per musica e danza. In Storia della letteratura italiana, hgg. E. Malato, Bd. 2: Il Trecento. Rom: 455-529. 